08,11

\title{
Формирование регулярных слоистых структур при твердотельных фазовых переходах с изменением концентрации
}

\author{
(C) А.А. Чеврычкина, Н.М. Бессонов, А.Л. Корженевский \\ Институт проблем машиноведения РАН, \\ Санкт-Петербург, Россия \\ ฯ E-mail: alekorzh@mail.ru
}

Поступила в Редакцию 1 апреля 2020 г.

В окончательной редакции 1 апреля 2020 г.

Принята к публикации 5 апреля 2020 г.

В рамках одномерной капиллярно-волновой модели рассчитана автоколебательная динамика межфазной границы и параметры периодической слоистой примесной микроструктуры, возникающей в результате твердотельного фазового перехода с изменением концентрации, происходящего при приложении подвижного градиента температуры.

Ключевые слова: твердотельные фазовые переходы, примеси, движущийся градиент температуры, капиллярно-волновая модель, расчет профиля периодических примесных сверхструктур.

DOI: 10.21883/FTT.2020.08.49609.074

\section{1. Введение}

Большая часть исследований по изучению процессов фазовых переходов (ФП) 1-го рода проводится в условиях, максимально приближенным к изотермическим. Тем не менее, в реальных экспериментах затруднительно полностью избавиться от температурных градиентов, наличие которых может существенно повлиять на характер ФП. Кроме того, в ряде методов выращивания кристаллов из растворов и расплавов наличие ненулевого градиента температуры $\nabla T$ (ГТ) является ключевым элементом методики [1]. Методика, в которой используется подвижный ГТ, кратко называется направленной кристаллизацией (НК). НК применяется при лабораторных исследованиях, преимущества которых состоят в том, что условия эксперимента задаются двумя независимыми параметрами (величиной $\nabla T$ и скоростью смещения изотерм $\left.V_{p}\right)$. Помимо этого, процесс НК является важной составляющей полного промышленного цикла выплавки металлов [2]. По сути, близкая физическая ситуация возникает и в ряде естественных геофизических и геохимических процессов, таких как охлаждение интрузивов, различные ФП в недрах Земли и т. п. [3].

Физическая картина процессов, сопровождающих НК, является сложной. Во-первых, перпендикулярно ГТ формируется макроскопический плоский фронт, разделяющий исходную и конечную фазу. Его текущее положение отслеживает движение изотермы, температура которой определяется точкой ФП. При этом на мезоскопическом масштабе геометрия фронта, вообще говоря, может оказаться неплоской из-за наличия морфологических неустойчивостей, наиболее известной из которых является неустойчивость Маллинза и Секерки [4]. Во-вторых, поскольку фронт находится в области температур гистерезиса $Ф П$, в его окрестности могут появляться зародыши другой фазы, что приводит к сложной топологии переходной двухфазной области.
В сплавах, обычно испытывающих ФП с изменением концентрации, в условиях НК, значение концентрации примеси на фронте зависит не только от его положения, но и от полной динамики межфазной границы, включающей эффект памяти. В том случае, когда динамика фронта не является стационарной, при его перемещении примесь откладывается в фазе-продукте неравномерно. Возникающую в ней пространственно-неоднородную примесную структуру можно затем зафиксировать, например, резко понижая температуру, прикладывая внешнее давление, сдвигающее точку перехода и т. п.

Естественно, возникает вопрос, нельзя ли целенаправленно управлять примесным профилем структуры продукта. Из сказанного выше очевидно, что для этого в первую очередь надо достаточно просто и надежно уметь рассчитывать концентрацию примеси на нестационарном фронте. Динамика последнего, в свою очередь, зависит от величины примесного торможения, которое определяется распределением примеси в окрестности межфазной границы.

В настоящей работе мы покажем, что система уравнений на координату морфологически устойчивого плоского фронта и поле концентрации примеси в определенной области управляющих параметров $\nabla T$ и $V_{p}$ допускает упрощение до решения одного нелинейного обыкновенного дифференциального уравнения типа обобщенного уравнения Рэлея, причем значение входящего в него коэффициента при старшей производной мало́. Важно, что в этой области параметров возникают осцилляции скорости межфазной границы, которые происходят в режиме релаксационных колебаний. Эти колебания индуцированы устойчивым предельным циклом на фазовой плоскости, обладающим очень простой формой. Наличие таких колебаний позволяет предложить методику управления параметрами периодических слоистых примесных структур в кристаллах за счет оптимального выбора режима НК. 
План изложения в нашей статье таков. В разделе 2 мы кратко приведем основные теоретические результаты для НК и экспериментальные факты, известные о слоистых структурах в металлических сплавах. В разделе 3 описана используемая в нашем анализе капиллярноволновая модель (КВМ), приведены ее уравнения движения в точной и приближенной форме, а также проведено сравнение значений наиболее существенных параметров для задач при НК из расплава и при ФП между твердыми фазами. В разделе 4 приведены результаты наших аналитических и численных расчетов динамики межфазных границ и примесных профилей концентрации, формирующихся при выборе оптимального режима НК для твердотельных ФП. В разделе 5 суммируются полученные результаты и кратко обсуждается возможность создания на их основе технологии, позволяющей создавать примесные периодические структуры заданного профиля.

\section{2. Экспериментальные факты и теоретические исследования, описывающие свойства периодических слоистых примесных структур и механизмы их формирования при направленной кристаллизации сплавов}

Экспериментально нестационарная динамика фронта была обнаружена при быстрой НК ряда металлических сплавов, в которых наблюдалось появление слоистых примесных структур (ставших известными как „banded structures") [5-8]. Теоретически на возможность осцилляций скорости границы расплав-кристалл в таком режиме впервые было указано в [9], где термодинамический анализ линейной устойчивости плоской границы был обобщен за счет введения неравновесного коэффициента распределения примеси $k(V)$, зависящего от скорости $V$. Более последовательный учет отклонения состояния материала на фронте от равновесного был проведен в [10]. Опираясь на эти результаты для плоского осциллирующего фронта, а также дополнительно учитывая возможность формирования в двухфазной области фронта, обладающего дендритной морфологией, в [8] был предложен феноменологический рецепт построения разрывного предельного цикла, качественно иллюстрирующий возможный сценарий происхождения периодических слоистых структур при быстрой НК.

Все эти исследования использовали модели с геометрически тонкими („sharp“) фронтами. Однако подобные модели не позволяют корректно вычислять силы примесного торможения фронтов, а лишь постулируют для них ту или иную зависимость от мгновенной скорости $V(t)$, заимствованную из предельно упрощенных микроскопических расчетов, выполненных для фронтов, движущихся с постоянной скоростью [11]. Полностью игнорируя тем самым эффекты памяти, такие модели не могут претендовать на сколько-нибудь надежное количественное описание динамики нестационарных режимов и порожденных ею пространственно-неоднородных структур.

Это обстоятельство, а также чисто математические трудности, возникающие как при попытках аналитического решения динамических задач со свободной границей (форма которой заранее неизвестна и должна находиться как часть решения), так и при численном моделировании, привели к тому, что в качестве конструктивной альтернативы им возник, и вскоре приобрел доминирующее значение, метод фазового поля (см., например, обзоры $[12,13])$. Уравнения движения в моделях с фазовым полем определяют динамику состояния материала в любой его точке, вследствие чего необходимость постановки дополнительных условий на подвижной межфазной границе отпадает.

Этот метод был использован и для решения задачи НК в сплавах [14,15]. Было показано, что при некоторых выбранных значениях параметров модели движение фронта расплав-кристалл становится осциллирующим за счет возникновения устойчивого предельного цикла в плоскости температура-скорость. Напомним, что идея о существовании в динамике НК предельного цикла лежала и в основе феноменологического сценария формирования периодических слоистых структур, предложенного в [8], и отчасти подтвержденного в [16]. Однако в [14-16] отмечалось, что полученная численно форма цикла и поведение фазовых траекторий отличаются от предсказанных в [8]. Было высказано предположение, что это происходит из-за отсутствия учета эффекта запаздывания примесного поля по отношению к движению фронта в [8].

В результатах работ $[14,15]$ отчетливо отразились как достоинства, так и недостатки, присущие общему методу фазового поля. С одной стороны, метод позволил воспроизвести основные качественные черты динамики формирования периодических примесных структур (ППС), наблюдаемые экспериментально. С другой, для установления общих параметрических зависимостей он требует большого количества повторных численных расчетов, проводимых на значительной части массива исходных параметров модели. При этом все равно сохраняется некоторая неуверенность в предсказаниях поведения модели для остальных значений параметров. Помимо этого, необходим дополнительный анализ при сопоставлении результатов многочисленных симуляций, что также снижает ценность метода.

По этим причинам, а также для выработки более глубокого понимания физики процессов НК в [17] была разработана новая капиллярно-волновая модель, сохраняющая наглядное представление о существовании разделяющей фазы поверхности, но в отличие от геометрической, имеющей конечную ширину. Эта модель, математически строго выведенная из базовой модели фазового поля, существенно упростила постановку задачи НК. Как следствие, ее применение позволило получить 
ряд новых аналитических результатов, описывающих динамику границ расплав-кристалл при НК разбавленных металлических сплавов [18-25].

В настоящей статье исследуется проблема описания динамики НК и расчета профилей концентрации примесных слоистых структур, формирующихся в результате превращений между двумя твердотельными фазами. Для этого мы применим модель, аналогичную использованной в $[23,24]$, но учтем в ней отличия от процесса НК из расплавов, описанные в следующем разделе.

\section{3. Уравнения движения и сравнение параметров капиллярно-волновых моделей твердотельных фазовых переходов с изменением концентрации и направленной кристаллизации сплавов из расплавов}

Сначала поясним, почему вообще капиллярноволновая модель (КВМ) способна эффективно описывать процесс НК для твердотельных ФП с изменением концентрации, хотя первоначально она была разработана для НК сплавов из расплавов [17].

При выводе КВМ был использован тот фундаментальный факт, что наличие межфазной границы нарушает континуальную трансляционную симметрию системы. По теореме Голдстоуна отсюда следует, что в такой системе должна появиться зона низкоэнергетических длинноволновых возбуждений. Физически этим возбуждениям соответствуют капиллярные волны на границе с малыми волновыми векторами (отсюда и происходит название КВМ).

Математический вывод КВМ состоит в том, что в исходной модели фазового поля удается проинтегрировать по всем ,жестким“ степеням свободы, отделенным в энергетическом спектре от зоны капиллярных волн щелью конечной величины и получить эффективный гамильтониан, зависящий только от „мягких“ степеней свободы, описывающих форму профиля $Z(x, y)$ межфазной границы. Дополнительные условия на значения материальных параметров, при которых такая процедура оказывается реально выполнимой, удовлетворяются как при НК из расплавов разбавленных металлических сплавов, так и при НК для многих твердотельных ФП (подробнее см. ниже).

Поскольку модель фазового поля определяется функционалом этого поля во всем трехмерном пространстве, а КВМ - функционалом только двумерного профиля $Z(x, y)$, то уже на этом этапе очевидно, что анализировать НК с помощью КВМ гораздо проще.

Кроме того, как было продемонстрировано нами в явном аналитическом виде в [23], ${ }^{1}$ при типичных значениях материальных параметров разбавленных сплавов,

\footnotetext{
${ }^{1}$ В формуле (18) нашей статьи [23] есть опечатка, в числителе последнего члена должна стоять буква $\gamma$ вместо буквы $\lambda$.
}

неустойчивость, ответственная за появление осцилляций скорости границы при НК, существует в гораздо более широкой области параметров на плоскости $\nabla T-V_{p}$, чем область морфологической неустойчивости Маллинза и Секерки. Это открывает возможность описывать НК в рамках еще более простой версии КВМ, оперирующей лишь с плоской межфазной границей. Уравнения движения для координаты $Z(t)$, параметризующей положение границы, и поля концентрации примеси $C(z-Z(t), t)$ могут быть тогда записаны в безразмерной форме (cp. c [24])

$$
\begin{gathered}
\frac{1}{p} \partial_{t} Z=F(Z)-\gamma \int_{-\infty}^{\infty} d z C_{E}(z-Z(t)) \\
\times\left[C(z, t)-C_{E}(z-Z(t)],\right. \\
\partial_{t} C=\partial_{z} d((z-Z(t))) \partial_{z}\left[C-C_{E}(z-Z(t))\right],
\end{gathered}
$$

где был произведен переход от размерных величин задачи, таких как заданный градиент температуры $\nabla T$, заданная постоянная скорость продвижения изотерм $V_{p}$, заданная концентрация примеси материала $C_{0}$, профиль равновесной концентрации примеси через межфазную границу $C_{E}(z)$, скачок концентрации примеси при ФП $\Delta C_{E}$, скрытая теплота ФП $L$, температура $Ф П T_{0}$, ширина границы $\delta$, наклон линии ФП на фазовой диаграмме $\frac{\partial T_{0}}{\partial C}$, максимальная скорость межфазной границы чистого вещества $V_{c}$, скорость перескока примесного атома в соседнюю ячейку $V_{d} \sim D_{0} / \delta$, и $D=D_{0} d(z / \delta)-$ коэффициент диффузии, к безразмерным переменным

$$
\frac{r}{\delta} \rightarrow r_{1}, \quad \frac{D t}{\delta^{2}} \rightarrow t, \quad \frac{2\left(C-C_{0}\right)}{\Delta C_{E}} \rightarrow C
$$

и независимым безразмерным параметрам

$$
\begin{gathered}
\gamma=\left(\frac{\Delta C_{E}}{2}\right)^{2} \frac{\rho \delta}{k T_{0}}, \quad \rho(T)=-\left(\frac{\partial T_{0}}{\partial C}\right) \frac{L}{T_{0}} \frac{1}{\Delta C(T)}, \\
p=\frac{V_{c}}{V_{d}}, \quad F(Z)=\frac{\delta^{3}}{k T_{0}}\left[\frac{T_{0}-T_{p}-g\left(Z-v_{p} t\right)}{T_{0}}\right], \\
g=\left(\frac{\delta^{3} L}{k T_{0}}\right)\left(\frac{\delta \nabla T}{T_{0}}\right), \quad d=\frac{D(z)}{D_{0}},
\end{gathered}
$$

где $k-$ константа Больцмана, $T_{p}-$ температура границы, движущейся с постоянной заданной скоростью изотермы $v_{p}$.

При записи уравнения диффузии (2) мы опустили инерционную поправку, учитывающую возможное отклонение от локального равновесия в объеме материала [26,27], чтобы максимально упростить сравнение полученных в настоящей статье результатов с условиями формирования слоистых примесных структур, недавно выведенными нами в аналитическом виде [23]. Такое упрощение никак не влияет на полученные нами ниже общие выводы.

Из (1), (2) видно, что в упрощенном „плоском“ варианте описание динамики КВМ сводится к расчету 
динамики материальной точки, взаимодействующей с одномерным полем концентрации примеси, распределенном в направлении, перпендикулярном границе.

Безразмерные уравнения (1), (2) удобно использовать для сравнения задач описания динамики НК при твердотельных фазовых переходах с изменением концентрации и при быстрой НК сплавов из расплавов. Мы видим, что несмотря на огромную разницу в численных значениях коэффициентов диффузии примесей в расплаве и в твердых фазах, с теоретической точки зрения эти задачи в основном различаются лишь выбором функции $d(z)$.

Действительно, при НК расплавленных сплавов очень хорошим приближением для $d(z)$ является $\theta(z)$ функция Хэвисайда (это приближение называют „односторонней“ моделью). В то же время при многих твердотельных ФП, для описания которых применима теория Ландау, перестройки кристаллической структуры малы и изменения диффузионных коэффициентов невелики. Поэтому для таких ФП хорошим приближением является модель с постоянным коэффициентом диффузии (известная как „двусторонняя“ модель), которую мы и будем подразумевать при дальнейшем изложении теории.

Что же касается экспериментального различия между твердотельной НК и НК из расплава, то оно, конечно, очень велико. В первую очередь, это обусловлено снижением коэффициента диффузии в твердых телах на несколько порядков по сравнению с жидкостью. Соответственно падает и значение диффузионной скорости $V_{d}$, например при $D_{0}=10^{-10} \mathrm{~cm}^{2} / \mathrm{s}$ имеем $V_{d}=10 \mu \mathrm{m} / \mathrm{s}$. Это означает возникновение куда более легких условий для экспериментальных измерений с высоким пространственно-временным разрешением даже при „быстрой“ твердотельной НК, в сравнении с ситуацией для расплавов.

Несмотря на это, вплоть до настоящего времени было проведено очень немного подобных экспериментов [28-30], причем во всех них были исследованы лишь твердотельные ФП с очень узкой гистерезисной областью (в безразмерных единицах им соответствуют очень малые значения $\gamma$ в (4)), что заведомо исключало формирование ППС (см. ниже). Вопрос об условиях их возникновения не обсуждался и в обширном теоретическом обзоре [31], где проводилось сопоставление общих методов и результатов для НК из расплавов и при твердотельных ФП.

Конечно, несмотря даже на столь радикальное упрощение, приводящее к модели (1), (2), задача аналитического описания НК все еще остается достаточно сложной в силу нелокальности взаимодействия примеси с осциллирующей границей, закон колебаний которой заранее неизвестен, а в свою очередь зависит от запаздывающей (имеющей „память“) динамики примесного поля.

Чтобы приближенно учесть это запаздывание и еще больше упростить КВМ, в [18] было выведено представление функции Грина для диффузионного уравнения в виде ряда, содержащего вклады операторов дифференцирования по времени сколь угодно высоких порядков. Это позволило представить точное решение диффузионного уравнения, в котором источник зависит от неизвестного положения границы $Z(t)$, в виде разложения по скорости $V(t)$ и ее временны́м производным всех порядков. Ясно, что $n$-ый член такого разложения, пропорциональный $d^{n} V / d t^{n}$, является проектором, т. е. дает ненулевой вклад в концентрацию примеси только на той части траектории положения границы, где она действительно движется с ускорением $n$-ой степени.

Как известно, релаксационные колебания выделяются среди остальных периодических движений тем, что на подавляющей части периода ускорения невелики, и лишь на очень малом отрезке времени вблизи точек поворота они резко возрастают [32]. Отсюда следует, что если мы интересуемся динамикой границы, предположительно движущейся в режиме релаксационных колебаний, то ряды для функции Грина и, следовательно, концентрации, можно оборвать, сохранив лишь члены пропорциональные $V$ и $\dot{V}$. Выражение для концентрации примеси принимает вид [24]:

$$
\begin{gathered}
C(\xi, t)=C^{(1)}(\xi, t)+C^{(2)}(\xi, t), \\
C^{(1)}(\xi, t)=\int_{-\infty}^{\xi} d \xi^{\prime} e^{-\left(\xi-\xi^{\prime}\right)} C_{E}^{\prime}\left(\xi^{\prime}\right), \\
C^{(2)}(\xi, t)=\frac{1}{2} \dot{v}\left(\frac{\partial^{2}}{\partial v^{2}}\right)\left\{\frac{\left[C^{(1)}(\xi, v)+C^{(1)}(\xi, 0)\right]}{v}\right\},
\end{gathered}
$$

где для вычисления значения концентрации на границе надо положить значение $\xi \equiv z-Z(t)=0$.

При этом неточность в вычислении временной зависимости концентрации $C(\xi=0, t)$ оказывается полностью сосредоточенной в пределах относительно малого промежутка времени $\tau$, которое граница проводит вблизи точек поворота. Подставив затем выраженное через $V$ и $\dot{V}$ значение $C(\xi, t)$ в уравнение силового баланса (1), определяющего траекторию $Z(t)$, мы сможем проверить, действительно ли полученное решение относится к классу релаксационных колебаний. ${ }^{2}$

Очевидно, что в результате такой подстановки уравнение (1) переходит в уравнение для нелинейного осциллятора, обладающего нелинейно зависящими от скорости силой трения $r(v)$ и массой $M(v)$

$$
M(v) \ddot{Z}+r(v)+g\left(Z-v_{p} t\right)-F=0,
$$

\footnotetext{
${ }^{2}$ Отметим, что предложенная нами схема расчета траектории $Z(t)$ отличается от классического метода расчета релаксационных колебаний, изложенного в [32]. В рамках последнего, при построении решения исходного нелинейного обыкновенного дифференциального уравнения, содержащего малый коэффициент $\epsilon$ при старшей производной, строят часть его решения до точки разворота, полагая $\epsilon=0$, т. е. решая редуцированное уравнение. Чтобы продолжить полученное решение за точку разворота, используют „,иипотезу скачка“ - физически разумное предположение, что энергия не успеет измениться при „мгновенном“ переходе к решению, полученному при том же значении „силь“, но для другой ветки изоклины. Более формально эта гипотеза подразумевает, что предел приращения смещения $\Delta Z=(\tau / 2) \ddot{Z}^{2} \Rightarrow 0$ при ускорении $\ddot{Z}$ стремящемся к бесконечности, времени скачка $\tau \Rightarrow 0$, но конечном значении их произведения, равном скачку скорости в точке разворота.
} 
где

$$
\begin{gathered}
r(v) \equiv \frac{v}{p}-f(v)+f(0), \\
M(v)=-\frac{1}{2} \frac{\partial^{2}}{\partial v^{2}}\left[\frac{f(v)+f(0)}{v}\right], \\
f(v) \equiv-\gamma \int_{-\infty}^{+\infty} d \xi C_{e}^{\prime}(\xi) C^{(1)}(\xi, v) .
\end{gathered}
$$

Учитывая, что при постоянной скорости $Z=v_{p} t$ имеем $r(v)=F$, удобно записать уравнение (8) для отклонения $h(t)=Z(t)-v_{p} t$ в виде обобщенного уравнения Рэлея

$$
M\left(v_{p}+\dot{h}\right) \ddot{h}+R\left(v_{p}+\dot{h}\right)+g h=0,
$$

где $R(v) \equiv r(v)-f(0)-\frac{v_{p}}{p}+f(p)$.

Несомненным достоинством уравнения (12) является то, что уже непосредственно из его вида ясно, что при $R^{\prime}(v)<0$ оно имеет по крайней мере один устойчивый предельный цикл. Отсюда, и из соотношений (5)-(7) следует, что наличие незатухающих осцилляций положения границы $Z(t)$ вокруг ее средней координаты $\bar{Z}=v_{p} t$ приведет к возникновению ППС в твердой фазе - продукте позади плоского фронта. Кроме того, поскольку функция $f(v)$, независимо от конкретного вида равновесного распределения примеси вблизи межфазной границы $C_{E}(z-Z)$, пропорциональна безразмерному множителю $\gamma$, то производная $R^{\prime}(v)=r^{\prime}(v)=\frac{1}{p}-f^{\prime}(v)$ может быть отрицательной, только если произведение $p \gamma$ достаточно велико. Поскольку неравенство $\gamma \sim \frac{\Delta T}{T_{0}} \ll 1$ (где $\Delta T$ - температурный интервал гистерезиса) выполняется, как правило, и при НК разбавленных сплавов, и для твердотельных ФП, вероятность появления ППС в этих двух случаях обусловлена разницей в типичных значениях параметра $p=\frac{V_{c}}{V_{d}}$. Нетрудно видеть, что из-за присущей твердотельным ФП малости значений коэффициентов диффузии, эта вероятность для них выше, т.е. ППС при таких ФП должны наблюдаться гораздо чаще, чем при НК разбавленных сплавов. Например, если взять значения $D \sim 10^{-10} \mathrm{~cm}^{2} / \mathrm{s}, \gamma \approx 10^{-2}, \delta \approx 10^{-7} \mathrm{~cm}$, то условие возникновения ППС удовлетворяется, если $V_{c} \gtrsim \frac{\gamma D}{\delta} \sim 0.1 \mu \mathrm{m} / \mathrm{s}$. Для очень многих твердотельных ФП максимальные скорости движения границ гораздо выше таких значений.

Типичные численные значения ГТ при таких переходах обычно намного меньше, чем при быстрой НК разбавленных расплавов, в которых наблюдались ППС: для последних величина ГТ оценивается как $\nabla T \sim 5 \cdot 10^{4} \mathrm{~K} / \mathrm{cm}[33]$, а при твердотельных ФП обычно используются $\nabla T \sim 5 \cdot\left(10^{1}-10^{2}\right) \mathrm{K} / \mathrm{cm}[1]$. При этом, из-за гораздо более низких значений скоростей границы $V \lesssim V_{d}$ и чисел Льюиса, роль процесса выделения скрытой теплоты (величина которой $L$ к тому же заметно меньше, чем в случае расплавов [1]) для этих ФП сильно подавлена, и наше модельное приближение заданного постоянного ГТ гораздо точнее, чем в случае
НК расплавов (ср. с обсуждением в [16] и приведенной там оценкой вклада этого процесса в уравнении (62)).

Чтобы рассчитать в явном виде динамику межфазной границы для твердотельных ФП, необходимо выбрать разумный профиль концентрации примеси $C_{E}(z)$ вокруг нее, а также использовать при расчете типичные для них численные значения материальных параметров, в соответствии с оценками, приведенными выше. Результаты наших расчетов приведены в следующем разделе.

\section{4. Автоколебательная динамика фазовой границы при твердотельных фазовых переходах с изменением концентрации примеси}

В настоящей статье мы ограничимся исследованием динамики того класса межфазных границ, для которых эффектами сегрегации примесей можно пренебречь. В этом случае профиль равновесного распределения примеси $C_{E}(z-Z)$ в окрестности границы меняется с расстоянием от нее монотонно. Поэтому в наших безразмерных расчетах мы использовали для ее моделирования следующую функцию

$$
\begin{aligned}
C_{E}(z-Z)= & \theta(Z-z) \exp (z-Z) \\
& +\theta(z-Z)[2-\exp (Z-z)] .
\end{aligned}
$$

Подставляя (13) в (11), находим функцию $f(v)$ в виде

$$
f(v)=-\gamma \frac{v+2}{(v+1)^{2}} .
$$

Заданное значение скорости изотерм $v_{p}=0.5$ мы выбрали внутри области существования колебательной неустойчивости плоской границы, определенной для „Двусторонней“ модели аналитически (см. формулу (21) в [23]), так, чтобы при типичных для быстрой НК значениях $g \sim 10^{-5}$ размер предельного цикла был мал, а его форма близка к овальной [24]. Для интересующих же нас твердотельных ФП, в соответствии с данными, приведенными в разделе 3 , естественно считать, что $g \sim 10^{-7}-10^{-8}$. Используя функции (13), (14) и подставляя указанные численные значения параметров в точные модельные уравнения (1), (2) или приближенное уравнение (12), находим временную зависимость положения границы $Z(t)$ и профиль концентрации примеси $C(z, t)$.

Так как дифференциальное уравнение (12) можно переписать в новых переменных $g t \Rightarrow \tau, g z \Rightarrow \tilde{z}$ в виде

$$
g M\left(v_{p}+\dot{h}\right) \ddot{h}+R\left(v_{p}+\dot{h}\right)+h=0,
$$

с малым множителем $g$ перед старшей производной, а не включенные в (15) вклады, пропорциональные производным $n$-го порядка, будут иметь коэффициенты $\sim g^{n-1}$, то может показаться, что вполне достаточно 


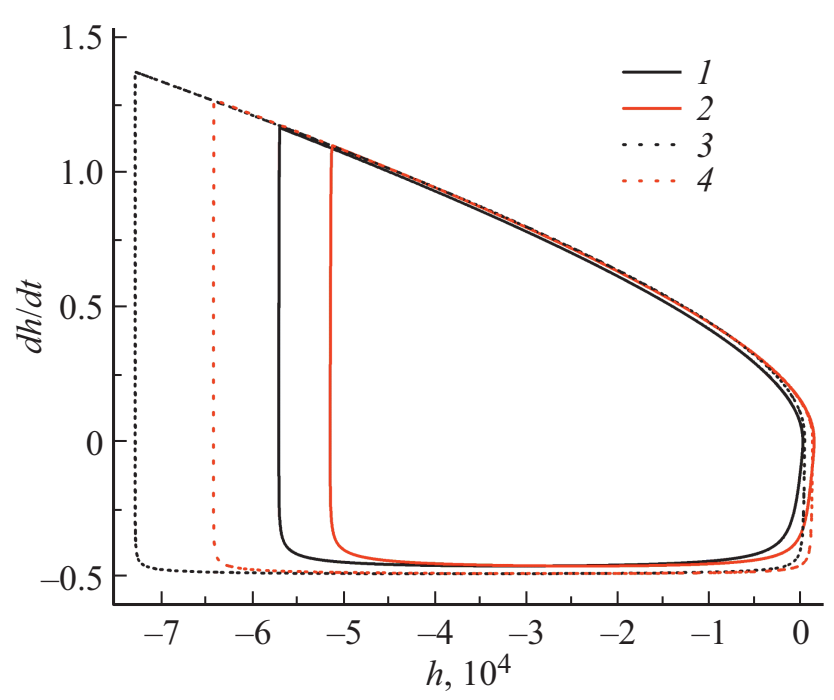

Рис. 1. Фазовые циклы для уравнений $(1-2)$ и (12) при $v_{0}=0.5, \gamma=0.01, p=100$. Линия 1 решение уравнения (12) при $g=7.5 \cdot 10^{-7}$, линия 2 решение уравнения $(1-2)$ при $g=7.5 \cdot 10^{-7}$, линия 3 решение уравнения (12) при $g=1 \cdot 10^{-7}$, линия 4 решение уравнения $(1-2)$ при $g=1 \cdot 10^{-7}$.

ограничиться исключительно решением уравнения (15). Однако надо учитывать, что ряд по степеням дифференциального оператора $\left(\frac{\partial}{\partial t}\right)$ является не сходящимся, а лишь асимптотическим, поскольку процесс диффузии примеси обладает памятью. Кроме того, как отмечалось в $[19,24]$, масса $M(v \rightarrow 0) \sim v^{-3}$, а „гипермассы“ $^{\text {“ }} M_{n-1}(v \rightarrow 0) \sim v^{3(1-n)}$. Таким образом, реальный параметр разложения ведет себя как $\epsilon \cong \frac{g}{v^{3}}$. При этом существенно, что в знаменателе стоит текущая скорость $v(t)$, а не экспериментально задаваемая постоянная $v_{p}$. В последнем случае в пределе $\epsilon \rightarrow 0$ можно было бы построить решение уравнения (15) с помощью классической теории для циклов разрывных автоколебаний [32]. Однако в случае уравнения (15) ситуация не является столь простой, поэтому необходимо сначала решить точные модельные интегро-дифференциальные уравнения (1), (2) в области параметров, где $\epsilon_{p} \equiv \frac{g}{v_{p}^{3}} \ll 1$, а затем выяснить, как количественно соотносятся эти решения с соответствующими решениями приближенного дифференциального уравнения (15). Предельные циклы, вычисленные из уравнений (1), (2) и (15) при $v_{p}=0.5$ и различных значениях $g$, приведены на рис. 1 .

Из рис. 1 видно, что с уменьшением значений градиента температуры $g$, циклы теряют овальную форму, и кроме того, циклы, вычисленные для нелинейного осциллятора (15), все лучше и лучше аппроксимируют их аналоги, полученные из точных уравнений (1), (2). Отсюда и без проведения дополнительных вычислений при еще меньших значениях $g \sim 10^{-8}$, требующих очень больших затрат машинного времени, ясно, что для большинства твердотельных ФП, проводимых при $\nabla T \lesssim 10^{2} \mathrm{~K} / \mathrm{cm}$, можно с достаточно хорошей практи- ческой точностью сразу пользоваться решениями более простого уравнения (15).

При этом, однако, нужно избежать ситуации, когда значения мгновенной скорости границы на каком-то интервале времени оказываются ниже, чем скорость верхней границы области морфологической неустойчивости Малинза и Секерки (так называемый „the limit of absolute stability“, $\left.V_{a s}\right)$. Если это произойдет и $V(t)<V_{a s}$, то слои формирующейся периодической примесной структуры потеряют свою плоскую форму. В таком случае применение одномерной КВМ становится неадекватным и для расчетов необходимо использовать ее более сложную версию.

Проиллюстрируем с помощью рис. 2, что подобная ситуация вполне возможна. На нем приведена зависимость минимального значения мгновенной скорости $v_{\min }(g)$ при фиксированной $v_{p}=0.5$.

Согласно результатам, полученным в [23], безразмерное значение $v_{a s}=4 \gamma$. Из рис. 2 видно, что оно достигается при $g_{*}=7 \cdot 10^{-7}$, для значений $g<g_{*}$ одномерная версия КВМ становится неприменимой. По этой причине, несмотря на очевидную падающую зависимость $v_{\min }(g)$ при $g \rightarrow 0$, вопрос о возможности достижения отрицательных значений $v(t)$ (т. е. появления обратного

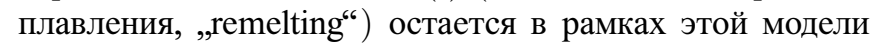
нерешенным и требует дальнейшего исследования.

Ясно также, что если по технологическим причинам необходимо добиваться формирования именно плоской слоистой структуры, то надо подбирать оптимальные значения по обоим управляющим параметрам $\nabla T$ и $V_{p}$. В частности, если выбрать значение $V_{p}=0.52$, то простые формы циклов, удовлетворяющие условию плоскости фронта, получаются при $g>g_{*}=3.05 \cdot 10^{-7}$, т.е. критическое значение $g_{*}$ снижается больше, чем в два раза по сравнению с примером, приведенным на рис. 2.

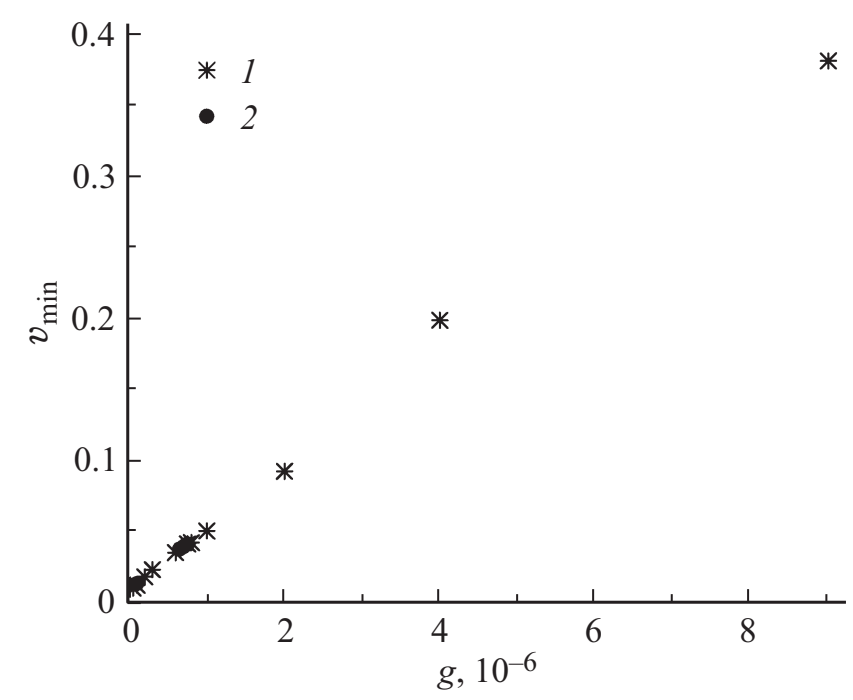

Рис. 2. Зависимость $v_{\min }$ от $g$ при $v_{0}=0.5, \gamma=0.01, p=100$. Точки 1 соответствуют уравнению (12), точке $2-(1-2)$. 
Отметим, что при численных значениях градиентов температуры, типичных для экспериментов с твердотельными ФП, динамика межфазных границ, как мы и предполагали выше, действительно имеет характер релаксационных колебаний. Такое поведение иллюстрируется рис. 3 , где приведены графики для $h(t), v(t), \ddot{h}$ и $\dddot{h}$.

В частности, как и ожидалось, заметные ускорения $\dot{h}$ и $\ddot{h}$ появляются лишь на временных интервалах $\tau$, очень малых по сравнению с полным периодом колебаний $T$, а именно $\tau / T \sim 10^{-4}$.

Рассчитать профиль концентрации в фазе-продукте можно либо из точных модельных уравнений (1), (2), либо подставляя решение приближенного уравнения (12) в уравнения (5-7). Соответствующие результаты приведены на рис. 4, $a, b$. Из их сравнения видно, что, хотя и имеется сравнительно небольшая, порядка $10 \%$, разница в полученных значениях длин пространственных периодов микроструктуры, сами формы профилей очень близки. Отсюда ясно, что с неплохой точностью для расчета профиля концентрации можно ограничиться решением более простых уравнений (5-8).

Грубую, но полезную оценку для ширины слоя $d=V_{p} T$ (где $T-$ период релаксационных колебаний $)$

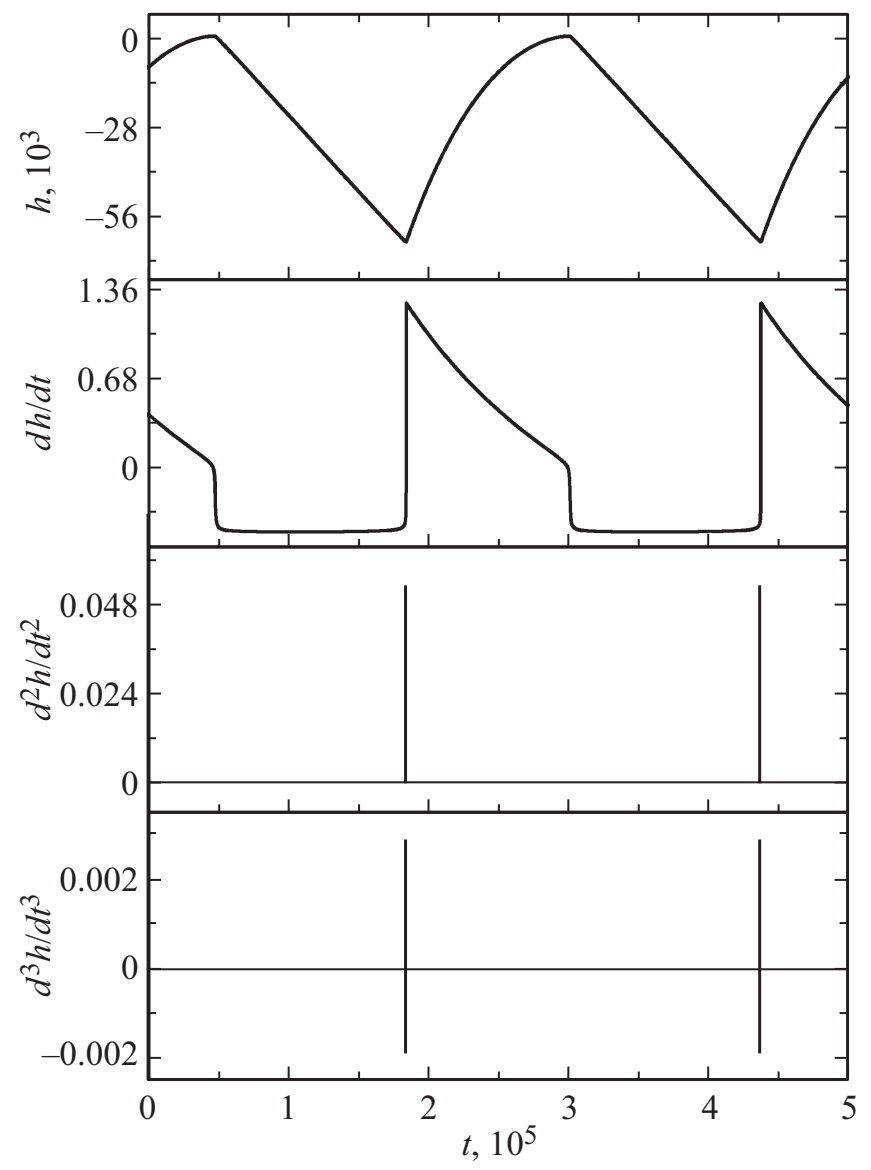

Рис. 3. Зависимости $h(t), \dot{h}(t), \ddot{h}(t), \dddot{h}(t)$ для уравнений $(1)-(2)$ при значениях параметров $v_{0}=0.5, g=1 \cdot 10^{-7}$, $\gamma=0.01, p=100$.

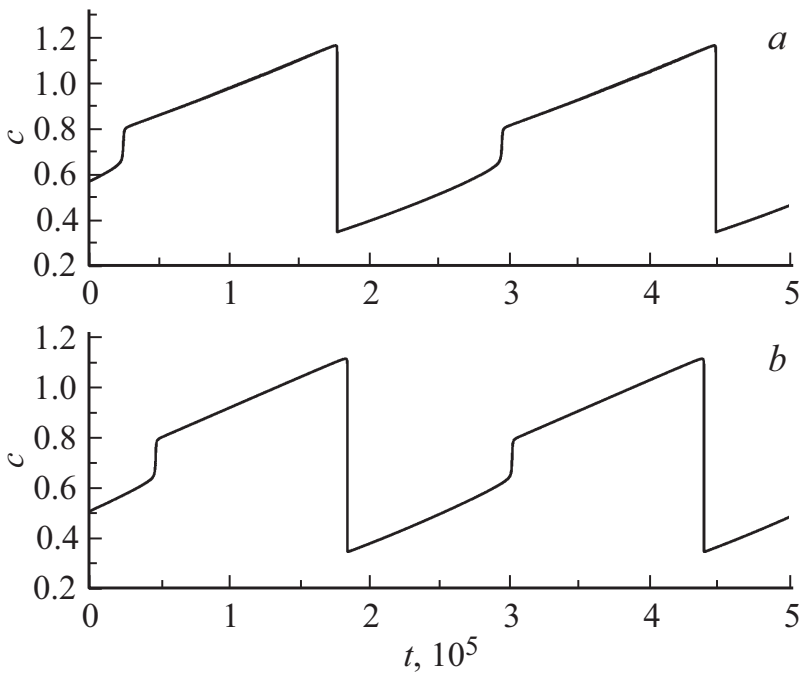

Рис. 4. Профиль концентрации а) уравнения (5-7), (12); $b)$ уравнения (1-2). Значения параметров $v_{0}=0.5, \gamma=0.01$, $p=100$.

можно получить, если сравнить твердофазный случай с НК из расплава. Для отношения $\frac{d_{s}}{d_{c}}$ имеем

$$
\frac{d_{s}}{d_{c}} \cong\left(\frac{g_{c}}{g_{s}}\right)\left(\frac{D_{s}}{D_{c}}\right),
$$

где индексы $c$ и $s$ соответствуют параметрам при быстрой НК из расплава и твердотельных ФП. Численно, отношение безразмерных градиентов в правой части порядка $10^{2}-10^{3}$. Отношение же диффузионных коэффициентов может изменяться в широких пределах. Для высокотемпературных ФП, имеющих значения $T_{0}$ недалеко от температур плавления, оно порядка $10^{-2}-10^{-3}$ [28-30], а для низкотемпературных обычно еще на несколько порядков меньше [34]. Таким образом, получаем $d_{s} \sim d_{c} \sim 1-10 \mu \mathrm{m}$ в первом случае и $d_{s} \ll d_{c}$ во втором.

\section{5. Заключение}

В рамках одномерной капиллярно-волновой модели мы рассчитали автоколебательную динамику межфазной границы и параметры периодической слоистой примесной микроструктуры, возникающей в результате твердотельного фазового перехода с изменением концентрации, происходящего при приложении подвижного градиента температуры. Отмечено, что выполнение условий, необходимых для появления таких автоколебаний, ожидается в существенно большем числе сплавов, чем при быстрой кристаллизации из расплавов. Было показано, что автоосцилляции границ имеют характер релаксационных колебаний, причем при типичных значениях материальных и управляющих параметров для расчета динамики достаточно решить обыкновенное 
нелинейное дифференциальное уравнение типа обобщенного уравнения Рэлея. Было также установлено, что при сравнительно небольших значениях градиента температур в промежутке $\nabla T \sim 50-150 \mathrm{~K} / \mathrm{cm}$, обычно использующихся в экспериментах, и скоростях изотерм в интервале $V_{p} \sim 1-10 \mu \mathrm{m} / \mathrm{s}$, можно подобрать значения этих управляющих параметров так, чтобы в кристаллепродукте реализовывалась слоистая микроструктура с заданным пространственным периодом. Его типичная численная оценка составляет порядка $1-10 \mu \mathrm{m}$ для высокотемпературных и имеет много меньшую, вплоть до наномасштабов, величину для низкотемпературных ФП.

Для построения более полной количественной теории слоистых микроструктур, формирующихся при твердотельных фазовых переходах, происходящих в условиях приложенного подвижного градиента температуры, необходимо в первую очередь исследовать влияние на них внутренних упругих напряжений. Кроме того, отдельного анализа заслуживает расчет возможного процесса зародышеобразования, сопутствующего движению межфазного фронта. Учет пространственного непостоянства градиента температуры не представляется принципиально важным из-за отмеченной выше малости характерных скоростей изотерм.

Отметим, что полученные для плоских слоистых микроструктур результаты во многом применимы и для задач, в которых движение изотерм не является одномерным в буквальном смысле (например, в задачах с цилиндрической или шаровой симметрией теплового поля).

Подводя итог, еще раз привлечем внимание к тому важному обстоятельству, что условия для экспериментальных исследований динамики твердотельных фазовых переходов в приложенных извне градиентов температуры гораздо благоприятнее, чем в случае затвердевания из расплавов. Поскольку и теоретический анализ в твердотельном случае требует лишь сравнительно простых расчетов, то разработка новой методики создания слоистых примесных микроструктур с заданным периодом представляется достаточно перспективной.

\section{Финансирование работы}

Работа выполнена при финансовой поддержке Российского научного фонда (проект № 19-19-00552).

\section{Конфликт интересов}

Авторы заявляют, что у них нет конфликта интересов.

\section{Список литературы}

[1] Х.С. Багдасаров, Е.И. Гиваргизов, Л.Н. Демьянец, В.А. Кузнецов, А.Н. Лабочев, А.А. Чернов. Современная Кристаллография / Под ред. Б.К. Вайнштейн. Наука, М. (1980). T. 3. 401 c.
[2] Р.У. Кан, П. Хаазен. Физическое металловедение. Металлургия, М. (1987). Т. 2. С. 178.

[3] В.К. Черепанова, А.Н. Черепанов, В.Н. Шарапов. Модели динамики фазовых превращений в магматических системах и металлических сплавах. Изд-во НГТУ, Новосибирск (2015). $243 \mathrm{c}$.

[4] W.W. Mullins, R.F. Sekerka. J. Appl. Phys. 35, 444 (1964).

[5] W.J. Boettinger, D. Schechtman, R.J. Schaffer, F.S. Biancaniello. Met. Trans. A 15, 55 (1984).

[6] M. Gremaud, M. Carrard, W. Kurz. Acta Met. Mater. 38, 2587 (1990).

[7] M. Gremaud, M. Carrard, W. Kurz. Acta Met. Mater. 39, 1431 (1991).

[8] M. Carrard, M. Gremaud, M. Zimmerman, W. Kurz. Acta Met. Mater. 40, 983 (1992).

[9] S.R. Coriell, R.F. Sekerka. J. Cryst. Growth 61, 499 (1983).

[10] M.J. Aziz, W.J. Boettinger. Acta Met. Mater. 42, 527 (1994).

[11] M.J. Aziz. J. Appl. Phys. 53, 1158 (1982).

[12] N. Provatas, K. Elder. Phase Field Methods in Materials Science and Engineering / Ed. Wiley-VCH. Wiley-VCH, Weinheim. (2010) 312 p.

[13] I. Steinbach. Phase-field models in materials science. Mod. Simul. Mater. Sci. Eng. 17, 7, 073001 (2009).

[14] M. Conti. Phys. Rev. E 56, R6267 (1997).

[15] M. Conti. Phys. Rev. E 58, 2071 (1998).

[16] A. Karma, A. Sarkissian. Phys. Rev. E 47, 513 (1993).

[17] A.L. Korzhenevskii, R. Bausch, R. Schmitz. Phys. Rev. E 83, 041609 (2011).

[18] A.L. Korzhenevskii, R. Bausch, R. Schmitz. Phys. Rev. Lett. 108, 046101 (2012).

[19] A.L. Korzhenevskii, R. Bausch, R. Schmitz. Phys. Rev. E 85, 021605 (2012).

[20] E. Babushkina, N.M. Bessonov, A.L. Korzhenevskii, R. Bausch, R. Schmitz. Phys. Rev. E 87, 042402 (2013).

[21] R.E. Rozas, A.L. Korzhenevskii, R. Bausch, R. Schmitz. Physica A 413, 394 (2014).

[22] R.E. Rozas, A.L. Korzhenevskii, J. Horbach. J. Phys. 28, 035001 (2016).

[23] А.А. Чеврычкина, Н.М. Бессонов, А.Л. Корженевский. ФTT 61, 1904 (2019).

[24] А.А. Чеврычкина, Н.М. Бессонов, А.Л. Корженевский. ФTT 61, 2122 (2019).

[25] A.A. Chevrychkina, N.M. Bessonov, A.L. Korzhenevskii, D.V. Alexandrov. Eur. Phys. J. Spec. Top. 229, 253 (2020).

[26] S.L. Sobolev. Int. J. Heat Mass Trans. 71, 295 (2014).

[27] P.K. Galenko, V. Ankudinov. Acta Mater. 168, 203 (2019).

[28] M. Vandyoussifi, H.W. Kerr, W. Kurz. Acta Mater. 45, 4093 (1997).

[29] M. Lima, W. Kurz. Met. Mater. Trans. A 33, 2337 (2002).

[30] A. Jacot, M. Sumida, W. Kurz. Acta Mater. 59, 1716 (2011).

[31] M. Asta, C. Beckermann, A. Karma, W. Kurz, R. Napolitano, M. Plapp, G. Purdy, M. Rappaz, R. Trivedi. Acta Mater. 57, 941 (2009).

[32] А.А. Андронов, А.А. Витт, С.Э. Хайкин. Теория колебаний. Физматлит, М. (1981). 568 с.

[33] S.C. Gill, W. Kurz. Mater. Sci. Eng. A 173, 335 (1993).

[34] Р.У. Кан, П. Хаазен. Физическое металловедение. Металлургия, М. (1987). Т. 2, С. 98.

Редактор Ю.Э. Китаев 